\title{
Involvement of FAN in TNF-induced apoptosis
}

\author{
Bruno Ségui, ${ }^{1}$ Olivier Cuvillier, ${ }^{1}$ Sabine Adam-Klages, ${ }^{2}$ Virginie Garcia, ${ }^{1}$ \\ Sophie Malagarie-Cazenave, ${ }^{1}$ Sophie Lévêque, ${ }^{1}$ Sylvie Caspar-Bauguil, ${ }^{1}$ Jérôme Coudert, ${ }^{3}$ \\ Robert Salvayre, ${ }^{1}$ Martin Krönke, ${ }^{4}$ and Thierry Levade ${ }^{1}$
}

\author{
${ }^{1}$ Institut National de la Santé et de la Recherche Médicale (INSERM) U466, Centre Hospitalier Universitaire Rangueil, \\ Toulouse, France \\ ${ }^{2}$ Institut für Immunologie, Christian-Albrechts Universität, Kiel, Germany \\ ${ }^{3}$ INSERM U28, CHU Purpan, Toulouse, France \\ ${ }^{4}$ Institut für Medizinische Mikrobiologie und Hygiene, Cologne, Germany
}

Address correspondence to: Thierry Levade, INSERM U466, CHU Rangueil, 1 Ave Poulhès, 31403 Toulouse, France. Phone: 33-561-32-20-60; Fax: 33-561-32-20-84; E-mail: levade@ rangueil.inserm.fr.

Received for publication October 6, 2000, and accepted in revised form May 21, 2001.

TNF- $\alpha$ is a pleiotropic cytokine activating several signaling pathways initiated at distinct intracellular domains of the TNF receptors. Although the C-terminal region is believed to be responsible for apoptosis induction, the functions of more membrane-proximal domains, including the domain that couples to neutral sphingomyelinase activation, are not yet fully elucidated. The roles of this region and of the associated adapter protein FAN (factor associated with neutral SMase activation) in the cytotoxic response to TNF have been investigated. We have now shown that stable expression in human fibroblasts of a dominant negative form of FAN abrogates TNF-induced ceramide generation from sphingomyelin hydrolysis and reduces caspase processing, thus markedly inhibiting TNFtriggered apoptosis. However, the cytotoxic responses to daunorubicin and exogenous ceramide remain unaltered, as do the TNF-induced p42/p44 MAPK activation and CD54 expression. Fibroblasts from FAN-knockout mice also proved to be resistant to TNF toxicity. These findings highlight the previously unrecognized role of the adapter protein FAN in signaling cell death induction by TNF. J. Clin. Invest. 108:143-151 (2001). DOI:10.1172/JCI200111498.

\section{Introduction}

Apoptosis is an essential process that regulates many aspects of normal and pathophysiological development of both vertebrates and invertebrates. The major biochemical pathways implicated in this process include the activation of caspases and of mitochondria-associated death events leading to the release in the cytosol of apoptogenic proteins such as cytochrome $c$ (cyt $c$ ) (1). Dysregulation of apoptosis may contribute to either degenerative diseases (if excessive) or cancers (if impaired) through cell transformation and development of resistance to chemotherapy. Major breakthroughs in understanding cell death mechanisms came from the elucidation of the signal transduction cascades initiated by the so-called death receptors and their respective ligands (2). These cell surface receptors belong to the TNF/nerve growth factor receptor superfamily that includes CD95/Fas (3).

TNF is a pleiotropic cytokine, mainly produced by monocytes and macrophages, that is implicated in several physiological and pathological conditions through the regulation of inflammatory and immune functions (4). It modulates growth and differentiation of different cell types and has antitumoral activity both in vivo and on many tumor cell lines (5). TNF effects are initiated by binding of the cytokine to specific, ubiquitously distributed, plasma membrane receptors: the p55 TNF receptor (TNFR-I or CD120a) and the p75 TNF receptor (TNFR-II or CD120b) $(5,6)$, which may oligomerize before ligand binding (7). Obliteration of individual receptor functions has indicated that, although TNFRs serve overlapping roles, CD120a mediates induction of cell death and defense against intracellular pathogens, whereas CD120b rather promotes inflammatory responses $(8-10)$.

The first step in signaling of these receptors is believed to be ligand-induced oligomerization of the receptor molecules, in particular through juxtaposition of their intracellular domains. Because these receptors are deprived of any intrinsic kinase activity, initiation of signaling occurs by recruitment of a network of cytosolic adapter proteins that associate with the cytoplasmic domains of TNFRs (6). These proteins then transduce TNF effects through activation of proteases, phospholipases, protein kinases, and transcription factors. Recent studies have emphasized the respective role of the intracellular domains of TNFRs (especially CD120a) and of various adapters. The C-terminal domain of CD120a contains an approximately 80-amino acid sequence, termed the death domain (DD), which has long been known to signal the cytotoxic effects of TNF $(11,12)$. This domain binds the p55 TNFR-associated DD protein (TRADD), which, in turn, recruits other DD motif-containing proteins, including the Fas-associated DD protein (FADD) and receptor-interacting protein (6). Thus, the protein com- 
plex initiated at the CD120a DD produces a signal that leads to apoptotic cell death through activation of initiator caspases (e.g., casp-8). The DD also mediates activation of an acidic sphingomyelinase (SMase), through TRADD and FADD (13-15), activation of p38 and p42/p44 mitogen-activated protein kinases (MAPK) (16), nuclear translocation of the transcription factor NF-KB, induction of IL-6 (17) and IL-8 (18) genes, and antiviral activity (12).

The N-terminal, membrane-proximal region of the intracellular domain of CD120a, upstream of the $\mathrm{DD}$, is able to associate with three proteins; namely, FAN (19), TRAP1 (20), and TRAP2 (21). FAN (factor associated with neutral SMase activation) is responsible for the stimulation of an SMase active at neutral $\mathrm{pH}$ through binding via a WD-repeat region to a short sequence (amino acids 309-319) of CD120a designated NSD (neutral SMase activation domain) (22). Increase in the latter enzyme activity results in the hydrolysis of the membrane phospholipid sphingomyelin (SM) and concomitant release of ceramide (Cer) (23). FAN-mediated generation of Cer is considered to activate the c-Raf- 1 signaling cascade through proline-directed protein kinases, and to possibly regulate TNF proinflammatory responses (13, 19, 24). The membrane-proximal half of CD120a also plays an important role in the induction of NO synthase (12), binding and activation of a phosphatidylinositol-4-phosphate 5-kinase (25), and translocation of mitochondria (26).

The sphingolipid Cer and its metabolites have recently emerged as important second messengers that may mediate a number of biologic effects, including induction of apoptosis (27-29). With regard to TNF signaling, different functions of Cer have been proposed (see above) that might be linked to distinct signal transduction pathways initiated at discrete regions of CD120a (13). Previous observations have shown that TNF can still promote SM hydrolysis in fibroblasts genetically deficient in acid SMase and can also promote their proliferation (30) or death (B. Ségui and T. Levade, unpublished observations), depending on their transformation status. These results, which suggested that a neutral, rather than acidic, SMase could be involved in transducing TNF effects, prompted us to investigate the role of FAN in the cell growth regulatory functions of TNF. Evidence is presented here that FAN is able to regulate TNF-induced apoptosis.

\section{Methods}

Reagents. Human TNF was obtained from PeproTech (Le Perray-en-Yvelines, France). Anti-human CD120a (clone htr-9) was purchased from Bachem (Voisins-leBretonneux, France), anti-active p42/p44 MAPK from Promega (Charbonnières, France), anti-p42/p44 MAPK from Santa Cruz Biotechnology Inc. (Santa Cruz, California, USA) and anti-CD54 coupled to fluorescein from Immunotech (Marseille, France).
Anti-casp-3 was obtained from D. Nicholson (PointeClaire, Quebec), anti-casp-8 from P. Krammer (Heidelberg, Germany) or G. Cohen (Leicester, United Kingdom), anti-Bid from X. Wang (Dallas, Texas, USA), anti-cyt $c$ from PharMingen (Le Pont de Claix, France), anti-cytochrome oxidase subunit II from Molecular Probes (Leiden, The Netherlands), and antiactin from Sigma (St. Quentin Fallavier, France).

[methyl- ${ }^{3} \mathrm{H}$ ]Choline chloride $(81 \mathrm{Ci} / \mathrm{mmol})$ and $\left[\gamma^{32} \mathrm{P}\right] \operatorname{ATP}(3,500 \mathrm{Ci} / \mathrm{mmol})$ were obtained from NEN (Paris, France). C2-Cer was supplied from Sigma, and daunorubicin from the NCI drug repository. The Escherichia coli strain expressing diacylglycerol kinase was a gift from D. Perry (Charleston, South Carolina, USA). All other reagents were of analytical grade.

Cell lines and transfections. Human SV40-transformed skin fibroblasts, derived from a normal individual (line Mav), were grown in DMEM containing Glutamax and $10 \%$ heat-inactivated FCS. The normal fibroblast cell line was transfected with the empty pcDNA3 plasmid or with pcDNA3 carrying a truncated version (encoding amino acids 703-917) of the FAN cDNA (31). Transfected cells were maintained in the presence of 0.2 $\mathrm{mg} / \mathrm{ml} \mathrm{G} 418$ as reported previously (31).

SV40-transformed fibroblasts from wild-type and FAN-knockout mice (32) were cultured under the same conditions as human fibroblasts. In some experiments, cells were incubated for 72 hours with a retroviral supernatant prepared as follows: GP+E86 cells were transfected by electroporation with a pLXSN vector carrying or not the full-length human FAN cDNA. After 72 hours of incubation in DMEM, the medium was harvested and filtered through $0.45-$ $\mu \mathrm{m}$-diameter filters.

U937 human leukemic cells (American Type Culture Collection, Rockville, Maryland, USA) were transfected using Lipofectamine (Life Technologies Inc., CergyPontoise, France) and cultured in RPMI 1640 containing $10 \% \mathrm{FCS}$ and $0.4 \mathrm{mg} / \mathrm{ml} \mathrm{G} 418$.

Metabolic labeling of cellular lipids. For SM quantitation, cells were incubated with $\left[\right.$ methyl $\left.{ }^{-3} \mathrm{H}\right]$ choline $(0.7$ $\mu \mathrm{Ci} / \mathrm{ml})$. After $48-72$ hours of incubation, cells were chased for 2 hours in fresh medium and then incubated at $37^{\circ} \mathrm{C}$ with or without TNF or daunorubicin. At the indicated times, cells were collected and sedimented at $4^{\circ} \mathrm{C}$ and cell pellets were immediately frozen at $-20^{\circ} \mathrm{C}$. Lipid extraction and analyses. Cell pellets were suspended in $0.6 \mathrm{ml}$ distilled water, and cells were disrupted at $4^{\circ} \mathrm{C}$ by brief sonication. Lipids were extracted, and $\left[{ }^{3} \mathrm{H}\right]$ choline-labeled SM was quantified as reported (30). Cer mass was measured as described elsewhere (33) using E. coli membranes as a source of diacylglycerol kinase and $\left[\gamma^{-32} \mathrm{P}\right]$ ATP. Radioactive Cer-1-phosphate was isolated by thin-layer chromatography using chloroform/acetone/methanol/acetic acid/water (50:20:15:10:5, by volume) as developing solvent.

Cell viability assays and morphological studies. Fibroblasts were plated in DMEM containing 10\% FCS in 24-well plates $(250,000$ cells per well). Cells were 

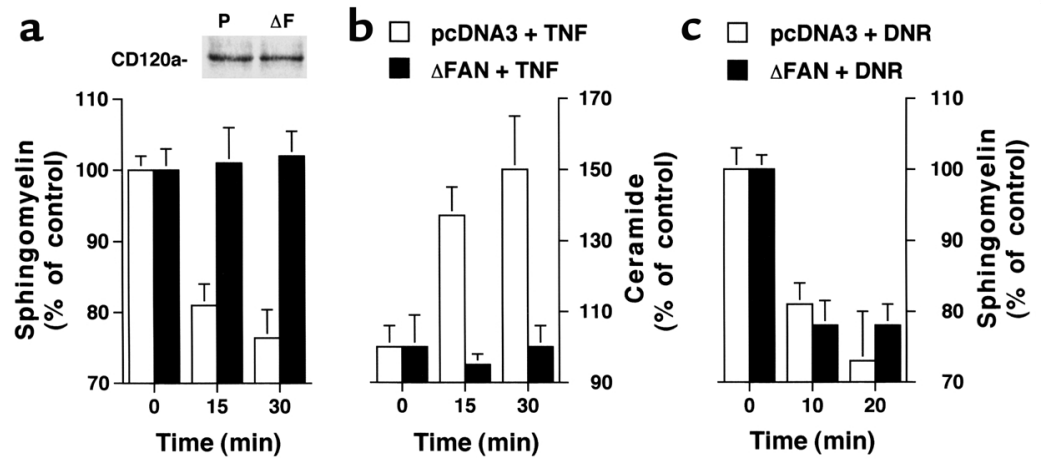

\section{Figure 1}

Alteration of the SM-Cer pathway induced by TNF in human fibroblasts expressing a truncated FAN. Human fibroblasts transfected with empty vector (pcDNA3) or a plasmid carrying a truncated FAN $(\triangle F A N)$ were labeled for 48 hours with $\left[{ }^{3} \mathrm{H}\right]$ choline. After a 2-hour chase, cells were incubated at $37^{\circ} \mathrm{C}$ in the presence of $50 \mathrm{ng} / \mathrm{ml}$ human TNF (a and $\mathbf{b}$ ) or with $1 \mu \mathrm{M}$ daunorubicin (DNR; $\mathbf{c}$ ). At the indicated time points, incubations were stopped, and SM levels (a and $\mathbf{c}$ ) were determined. Cer levels (b) were determined using diacylglycerol kinase. SM and Cer levels are expressed as percentage of the value observed before treatment. All values correspond to the mean \pm SE of three independent experiments. Inset: Expression of CD120a in fibroblasts transfected with the empty vector $(P)$ or a vector encoding the mutated FAN $(\Delta \mathrm{F})$.

treated with the indicated stimuli (exogenous Cer was delivered as an ethanolic solution) and further incubated at $37^{\circ} \mathrm{C}$ for the indicated times. Cell viability was estimated directly on culture plates by MTT assay or by coincubation with propidium iodide $(5 \mu \mathrm{M})$ and Syto-13 $(0.6 \mu \mathrm{M})(31)$. The percentage of apoptotic cells (having a condensed and fragmented nucleus), including postapoptotic cells (characterized by nuclear fragmentation, cell condensation and propidium iodide staining), was evaluated by counting cells ( 100 cells in at least three different fields for each well) under a Leica fluorescence-equipped inverted microscope. Flow cytometric analyses were performed on cells detached from the dishes using PBS containing $5 \mathrm{mM}$ EDTA on a FACScan (Becton Dickinson, Le Pont de Claix, France) cytometer.

$N F-\kappa B$ activity. Activation of NF- $\kappa B$ was analyzed by electrophoretic mobility shift assay. Preparation of nuclear extracts and binding reactions were performed as described previously (34).
Fluorogenic DEVD cleavage enzyme assay. After incubation with TNF, cells were sedimented, and casp-3 like activity was assessed using Ac-DEVD-AMC (Bachem) as described elsewhere (35).

Preparation of mitochondria and Western blotting analyses. Mitochondrial preparations were carried out as described previously (35). For Western blot analyses, equal amounts of proteins were separated on 7.5 or $15 \%$ SDS-PAGE (35).

\section{Results}

FAN mediates TNF-induced neutral SMase but not $p 42 / p 44$ MAPK activation. We have previously shown that in untransformed human fibroblasts, TNF promotes SM hydrolysis through a non-lysosomal SMase (30). Similarly, in human SV40-transformed fibroblasts, TNF induced a decrease in intracellular SM levels which started at 5 minutes and peaked at $30 \mathrm{~min}$ utes (with a $24 \pm 4 \%$ reduction); SM levels returned to baseline by 60 minutes (data not shown). This SM cycle was accompanied by the concomitant generation of Cer (50\% maximal increase at 15-30 minutes). Both the kinetics and amplitude of the responses were comparable to those seen in untransformed fibroblasts (30). To investigate the potential role of neutral SMase in TNF effects, we stably expressed a dominant negative, truncated form (amino acids 703-917) of the adapter protein FAN in human SV40-transformed fibroblasts (31). As shown in Figure 1, a and b, expression of the truncated form of FAN did not alter the levels of CD120a, yet it completely inhibited the TNF-induced SM degradation and Cer production compared with cells transfected with an empty vector. In contrast, cells expressing the mutated FAN responded normally to another inducer of the SM cycle, which activates a neutral SMase, the anthracycline daunorubicin (36), by undergoing SM turnover (Figure 1c). This suggested that the stimulation of neutral SMase by TNF, but not by the anthracycline, is regulated by FAN.

\section{Figure 2}

TNF-induced activation of p42/p44 MAPK in human fibroblasts. Human fibroblasts transfected with empty vector (pcDNA3) or a vector encoding the truncated FAN ( $\triangle \mathrm{FAN})$ were incubated for the indicated times in the presence or absence of human TNF $(50 \mathrm{ng} / \mathrm{ml})$. Expression of activated (dually phosphorylated; active MAPK) or total MAPK was visualized by Western blot (a). The data are representative of three independent experiments. (b) Quantification of p42/p44 MAPK activation by ImageQuant software (Molecular Dynamics, Sunnyvale, California, USA) $(n=3)$. The data for active MAPK were normalized to those for total MAPK.

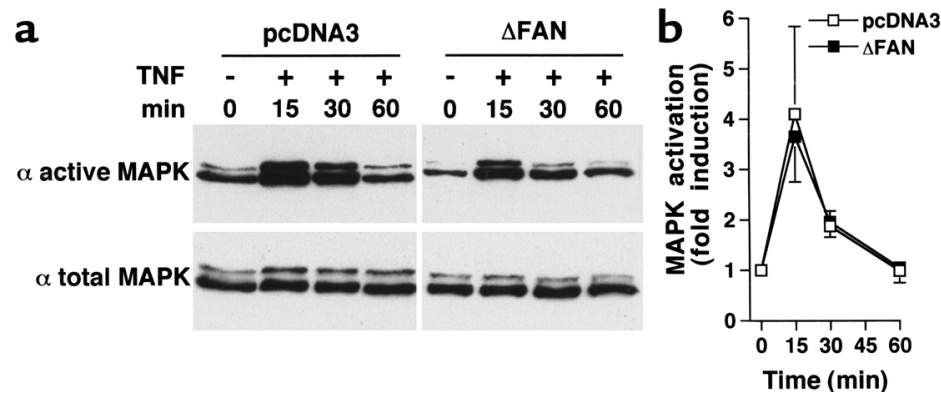



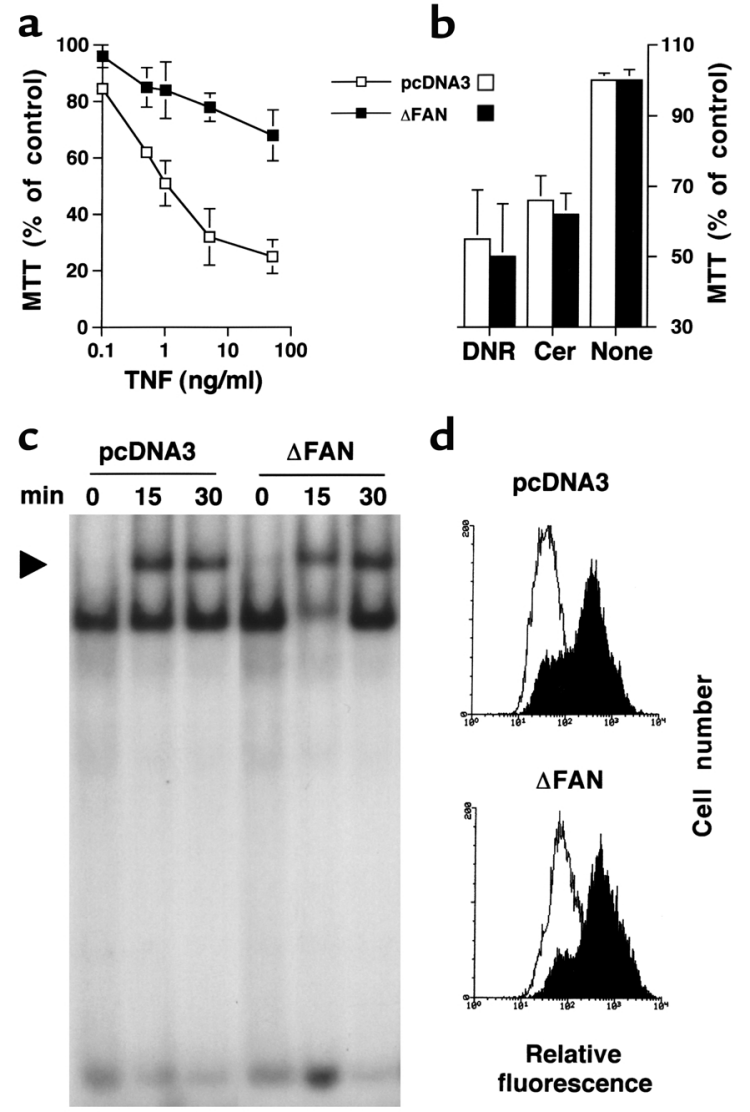

d

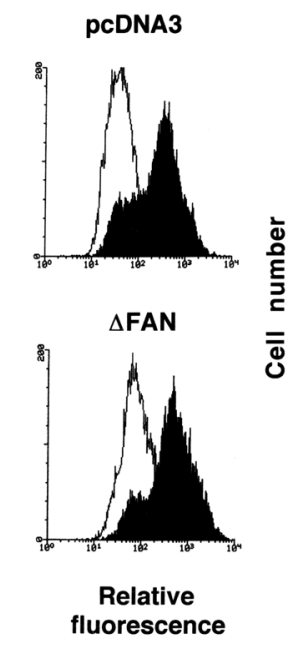

Figure 3

TNF-induced cell death, but not NF-KB activation or CD54 expression, is impaired in human fibroblasts expressing a truncated FAN. ( $\mathbf{a}$ and $\mathbf{b}$ ) Toxicity of TNF, daunorubicin, and Cer on human fibroblasts. Fibroblasts transfected with empty vector (pcDNA3) or a vector encoding the truncated FAN $(\triangle F A N)$ were treated for 24 hours with the indicated concentration of TNF (a) or $1 \mu \mathrm{M}$ daunorubicin (DNR) or $25 \mu \mathrm{M} \mathrm{C2-Cer} \mathrm{(Cer)} \mathrm{(b).} \mathrm{For} \mathrm{the} \mathrm{experiments} \mathrm{with} \mathrm{TNF}$ and Cer, $50 \mu \mathrm{g} / \mathrm{ml}$ of cycloheximide was added to the medium. Cell viability was estimated by the MTT test (mean \pm SE of three independent experiments performed in duplicate). (c) TNF-induced nuclear translocation of NF-KB in human transformed fibroblasts. pcDNA3 and $\triangle F A N$-transfected cells were treated for the indicated times with TNF $(50 \mathrm{ng} / \mathrm{ml})$. Nuclear extracts were incubated with a NF- $\kappa B$ oligoprobe and analyzed by a gel shift assay. The arrow indicates the retarded NF-KB-probe complex. (d) TNF-induced CD54 expression by human fibroblasts. Cells were incubated for 24 hours in the presence (filled histograms) or absence (open histograms) of TNF (100 ng/ml). CD54 expression was assessed by flow cytometry. Similar data were obtained in two additional independent experiments and using $50 \mathrm{ng} / \mathrm{ml} \mathrm{TNF}$.

The Cer generated via a neutral SMase has been proposed to mediate the activation of $\mathrm{c}-\mathrm{Raf}-1$, and subsequently the p42/p44 MAPK $(37,38)$. However, targeted disruption of FAN in mice has shown that FAN is not required for $\mathrm{p} 42 / \mathrm{p} 44$ MAPK activation (32). Accordingly, TNF was found to activate MAPK independently of FAN function. Both kinetics and amplitude of p42/p44 MAPK activation were similar in empty vector-transfected cells and cells expressing the dominant negative form of FAN (Figure 2). This indi- cates that in human fibroblasts, expression of the truncated FAN mimics its deficiency in murine fibroblasts with regard to neutral SMase and MAPK activation.

FAN regulates TNF-induced cytotoxicity but not $C D 54$ expression. In light of our showing that FAN is implicated in cell death induction by CD40 (31), a member of the TNFR superfamily, the sensitivity of control and mutant human SV40-transformed fibroblasts to the cytotoxic action of TNF was investigated. Previous work has also demonstrated that TNF incubation of human transformed fibroblasts with cycloheximide results in apoptosis (39). Under these conditions, TNF exhibited a dose-dependent cytotoxic effect on empty vector-transfected cells (Figure 3a). Overexpression of the FAN dominant negative form in these cells strongly inhibited cytotoxicity at each TNF concentration tested (Figure 3a). Yet, when the cells were challenged with daunorubicin or $\mathrm{C} 2$-Cer, they were as sensitive as their normal counterparts (Figure 3b).

To further evaluate the consequences of the expression of a truncated FAN on the TNF responses, other biologic activities of this cytokine on fibroblasts were assessed. Figure $3 \mathrm{c}$ shows that nuclear translocation of the NF- $\mathrm{KB}$ transcription factor, a well-established effect of $\operatorname{TNF}(5,6)$, appeared unaffected by the expression of the mutated FAN. TNF is also known to stimulate on fibroblasts the expression of adhesion molecules such as CD54 (40). As illustrated in Figure 3d, control and mutated FAN-expressing cells similarly responded to TNF by increasing the level of cell surface CD54.

To support the above observations made by using human cells expressing a dominant negative FAN, experiments were repeated on transformed fibroblasts derived from mice homozygous for a FAN-null mutation (32). Although FAN-deficient and wild-type murine fibroblasts exhibited a similar loss of cell viability upon daunorubicin treatment (Figure 4a) or after incubation with C2-Cer (data not shown), TNF toxicity was inhibited in $\mathrm{FAN}^{-/-}$cells (Figure $4 \mathrm{~b}$ ). Similar dosedependent responses were obtained by using either human or murine TNF, indicating that the effects are mediated by CD120a (5). Of note, preincubation of murine FAN-deficient fibroblasts with a retroviral vector encoding the full-length FAN led to restoration of their sensitivity to TNF (Figure 4c), further supporting a role of FAN in the cytotoxicity of TNF.

To extend our observations further to a cell lineage other than fibroblasts, we investigated the effects of the truncated FAN on the TNF-induced cytotoxicity on U937 human myeloid leukemic cells, a TNF-responsive cell line $(13,27)$. Although TNF treatment of control U937 for 24 hours decreased cell viability to $80 \pm 5 \%$, it reduced that of deleted FAN-transfected U937 cells to only $94.7 \pm 5 \%(n=4)$, suggesting that FAN can also regulate TNF-induced cytotoxicity in myeloid cells.

FAN regulates TNF-induced apoptosis. To characterize the role of FAN in the cell death process induced by TNF, the type of cell death was first investigated morphologically. Syto13/propidium iodide staining of 


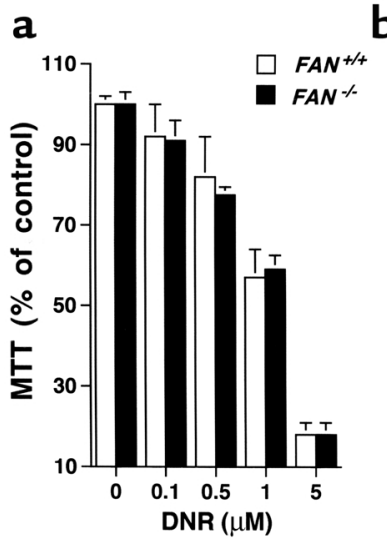

b

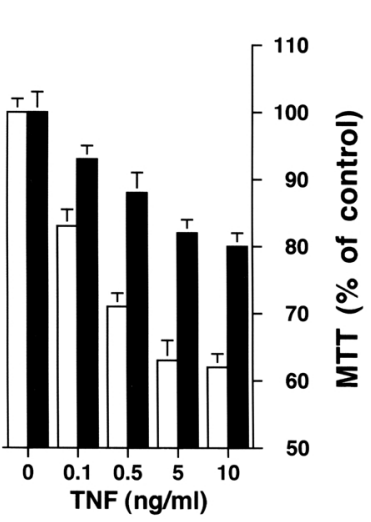

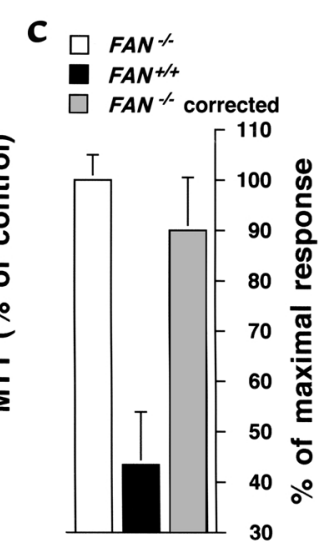

Figure 4

TNF-induced cell death is impaired in fibroblasts from FAN-deficient mice. SV40-transformed fibroblasts from wild-type $\left(F A N^{+/+}\right)$or FAN-knockout $\left(F A N^{-/-}\right)$mice were incubated for 24 hours in the presence of the indicated concentrations of daunorubicin (DNR) (a), or for 72 hours under serum-free conditions with the indicated concentrations of human TNF and without cycloheximide (b). (c) Murine FAN-deficient fibroblasts were preincubated for 72 hours in the presence of an empty retroviral vector $\left(F A N^{-/-}\right)$or a retroviral vector carrying the full-length FAN $\mathrm{CDNA}\left(F A \mathrm{~N}^{-/-}\right.$corrected) and were then treated for 72 hours with $10 \mathrm{ng} / \mathrm{ml}$ of human TNF. Viability of $F A N^{-1-}$ and $F A N^{-1-}$ corrected cells was assessed by $\mathrm{MTT}$ assay (mean \pm SE of three independent experiments) and expressed as percentage of the response observed in $\mathrm{FAN}^{+/+}$cells (see $\mathbf{b}$ ).

TNF in human SV40-transformed fibroblasts was examined. Preincubation of the cells in the presence of the pan-caspase inhibitor $z$-VADfmk abrogated the TNF-induced toxicity on transformed fibroblasts (cell viability averaged $30 \pm 5 \%$ and $95 \pm 5 \%$ in the absence and presence of $10 \mu \mathrm{M}$ z-VAD-fmk, respectively; $n$ $=3$ ) as well as the proteolytic cleavage of the effector casp-3 and casp-7 (data not shown).

These data indicated the essential role of caspases in the TNF-induced apoptosis of human transformed fibroblasts. A more detailed analysis of caspase activation further substantiated the role of FAN in the proteolytic cascade initiated by TNF. Indeed, determination of the global casp-3-like activity with the fluorogenic tetrapeptide substrate AcDEVD-AMC, which corresponds to the cleavage site found in numerous casp-3 and -7 targets, showed that expression of the truncated FAN

human fibroblasts treated in the presence of cycloheximide indicated that TNF addition resulted in an increased number of apoptotic cells, i.e., cells having a reduced size and a condensed and fragmented nucleus (Figure 5a). Under these conditions, the proportion of apoptotic cells was considerably lower in fibroblasts expressing the truncated FAN than in control fibroblast cultures (Figure 5a). In contrast, daunorubicin triggered apoptosis equally well in both types of cells (Figure $5 \mathrm{~b}$ ). The protection conferred by the dominant negative form of FAN against the cytocidal effect of TNF could also be observed after incubation of the cells with TNF in the absence of cycloheximide but under serum deprivation conditions (Figure $5, \mathrm{c}$ and d).

Because CD120a is a well-known death receptor able to activate the caspase cascade $(2,6)$, the implication of these proteases in the apoptotic cell death induced by

\section{Figure 5}

TNF-induced apoptosis is impaired in human fibroblasts expressing a truncated FAN. (a and $\mathbf{b}$ ) Human fibroblasts transfected with empty vector ( $p c D N A 3$ ) or a vector encoding the truncated FAN $(\triangle \mathrm{FAN})$ were treated for 24 hours in the presence or absence of 50 $\mu \mathrm{g} / \mathrm{ml}$ of cycloheximide (CHX) and $50 \mathrm{ng} / \mathrm{ml}$ of human TNF (a), or in the presence of the indicated concentration of daunorubicin (DNR) (b). Apoptotic cells were counted using Syto13/propidium iodide staining (mean $\pm \mathrm{SE}$ of three independent experiments). (c and d) Time course of TNF-induced apoptosis in human fibroblasts. Cells were incubated in a serum-free medium in the presence or absence of $50 \mathrm{ng} / \mathrm{ml} \mathrm{TNF}$, and apoptotic cells were counted at the indicated times (the data are from a triplicate experiment, representative of three independent experiments). potently inhibited TNF-induced caspase processing, either in the absence (Figure 6a) or presence (Figure $6 \mathrm{~b}$ ) of cycloheximide. This effect on casp-3-like activity was further demonstrated by Western blot analysis of individual caspases. As illustrated in Figure $6 c$, in cells expressing the dominant negative form of FAN, the cleavage of casp- 3 triggered by TNF (i.e., the
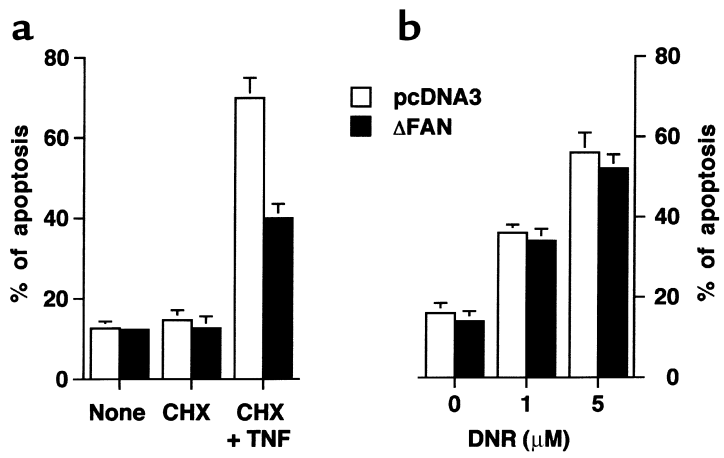

C

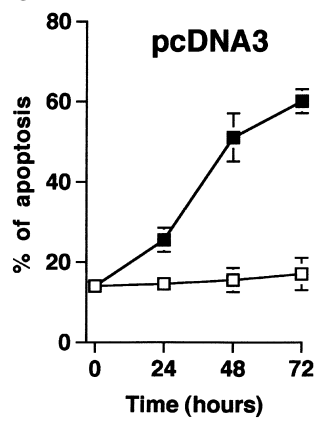

d

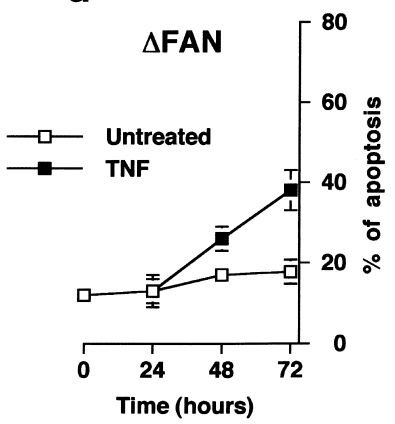


a b

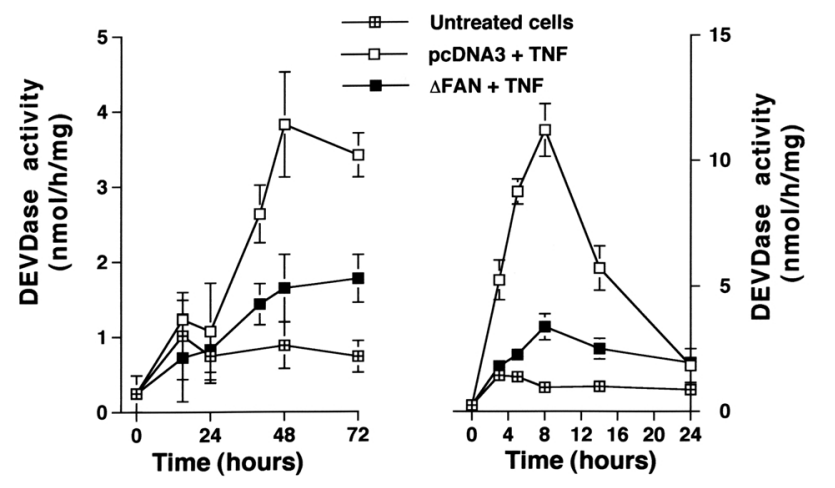

C

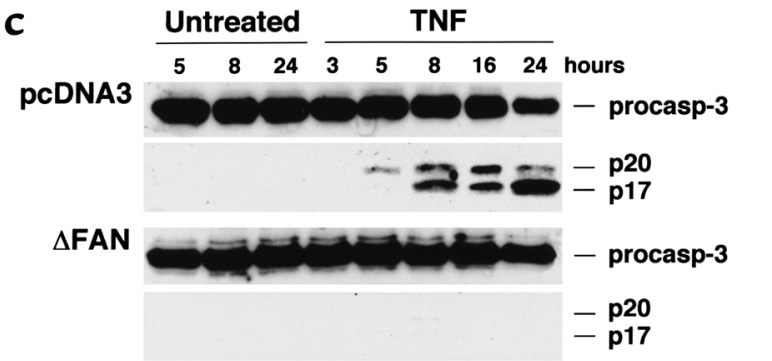

d

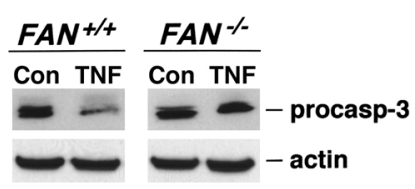

Figure 6

Caspase- 3 activation is impaired in fibroblasts expressing a truncated FAN and in FAN-deficient murine cells. SV40-transformed fibroblasts transfected with empty vector ( $p c D N A 3)$ or a vector encoding the truncated FAN $(\triangle F A N)$ were incubated in the presence or absence ofTNF $(50 \mathrm{ng} / \mathrm{ml})$, either under serum-free conditions (a) or in medium containing $1 \%$ serum and $50 \mu \mathrm{g} / \mathrm{ml}$ of cycloheximide (b and $\mathbf{c}$ ). At the indicated times, cells were harvested and DEVDase activity was determined ( $\mathbf{a}$ and $\mathbf{b}$; mean \pm SE of three independent experiments), and casp-3 processing was analyzed by Western blotting (c). Migrations indicated are procasp-3 (p32), cleavage intermediate p20, active subunit $\mathrm{p} 17$. (d) Fibroblasts from $F A N^{-1-}$ and wild-type mice were treated with TNF for 48 hours in serum-free medium, and casp-3 processing was assessed by immunoblotting (note that the antibody does not recognize cleaved forms in this murine cell line). Data are representative of three independent experiments.

decrease in the procaspase form and appearance of p20 and p17 forms) was almost completely suppressed. Similar findings were observed for the processing of the effector casp-7, and in the absence of cycloheximide under serum starvation conditions (data not shown). In the presence of TNF and cycloheximide, cleavage of the initiator casp- 8 was also seen (Figure 7a), which occurred quite concomitantly to that of the effector caspases. This cleavage was accompanied by the disappearance of Bid, a member of the Bcl-2 family known as a substrate of casp-8 (data not shown). Progressive release of cyt $c$ was also seen in cytosolic, mitochondria-free extracts from TNF-treated control cells (Figure 7b). Processing of both casp-8 and Bid, as well as the release of cyt $c$ in the cytosol, was considerably reduced in fibroblasts expressing the mutated FAN, further supporting the role of FAN in the apoptotic cascade initiated by TNF with a site of action lying upstream of mitochondria. Finally, the casp-3-like activity measured with the fluorogenic substrate Ac-DEVD-AMC was found to be decreased by $68 \%$ in FAN-deficient murine fibroblasts compared with cells from wild-type mice. Accordingly, TNFinduced casp-3 and casp- 8 cleavage was impaired in $F A N^{-/-}$cells (Figures $6 \mathrm{~d}$ and $7 \mathrm{~b}$ ).

\section{Discussion}

TNF elicits a wide range of biologic activities, but the precise elements in its receptors that mediate these different effects are not yet fully characterized. Although the cytocidal effects of TNF have long been thought to be signaled by the so-called DD of CD120a, this study demonstrates that a more membrane-proximal region of the receptor also participates, via recruitment of the FAN protein and possibly generation of Cer, in cell death induction.

FAN and TNF-induced apoptosis. Both extracellular and intracellular regions of TNFRs contain essential motifs for transducing the activities of TNF. Of crucial importance is the cytoplasmic part that interacts with various proteins, mediating different biologic responses. The functional significance of the intracellular domain of CD120a was demonstrated by expression of truncated mutants $(11,12)$. Because of the well-established cytotoxic effect exerted by TNF on numerous cell lines, a lot of attention has been paid to the cytoplasmic sequences of CD120a that mediate induction of cell death. So far, the only known region required for this activity is the DD, extending from amino acid 326 to amino acid 413 (12). Indeed, deletions of this region abolished the cytotoxic action of TNF on murine L929 cells (see also ref. 22). However, ablation of the cytoplasmic sequence spanning from amino acid 212 to amino acid 326 , but not a truncation of amino acids $212-308$, led to a reduction of approximately $50 \%$ of the TNF-induced cell death, suggesting that a more membrane-proximal domain than the DD is also implicated in the cytotoxic effect of TNF. According to Goeddel's studies, this domain covers the region termed the NSD (amino acids 309-319), which binds to the FAN protein $(19,22)$. Consistent with these observations, the present findings demonstrate that the apoptotic effect of TNF requires a signal emanating from a membrane-proximal region of CD120a, which is transduced by FAN. This tenet is supported by three lines of evidence. First, expression of a dominant negative form of FAN in human fibroblasts significantly reduces TNF-induced cell death. Second, fibroblasts from FAN-deficient mice are less susceptible to TNF cytotoxicity than are their normal counterparts. Third, transduction of murine FAN-deficient cells with a viral vector carrying human FAN cDNA restores their sensitivity to TNF. Although previous 
observations showed that the NSD by itself is unable to mediate the cytotoxic response (22), the present results demonstrate that this particular domain provides a signal concurring with those emanating from the DD for a full cell death induction, which well agrees with Goeddel's data (12).

That intracellular sequences of CD120a other than the DD contribute to cytotoxicity is not completely unexpected. The membrane-proximal part (amino acids 202-304) of the receptor exerts an important function in TNF cytotoxic signaling by allowing mitochondria to redistribute and cluster in the perinuclear region (26). In addition, the FAN-binding site of CD120a has recently been involved in other TNF responses, such as actin polymerization in macrophages (41) or induction of c-Raf-1 kinase activity (38). Finally, induction of cell death by CD40, another member of the TNFR superfamily, relies on the association of FAN to an intracellular portion of the receptor that shares some homology with the NSD (31).

How FAN regulates TNF-induced cell death. We show here that FAN controls an apoptotic program characterized by classical morphological alterations and caspase processing. The possibility that FAN affects this TNF response in an unspecific manner is unlikely, as (a) experiments on murine cells with human TNF

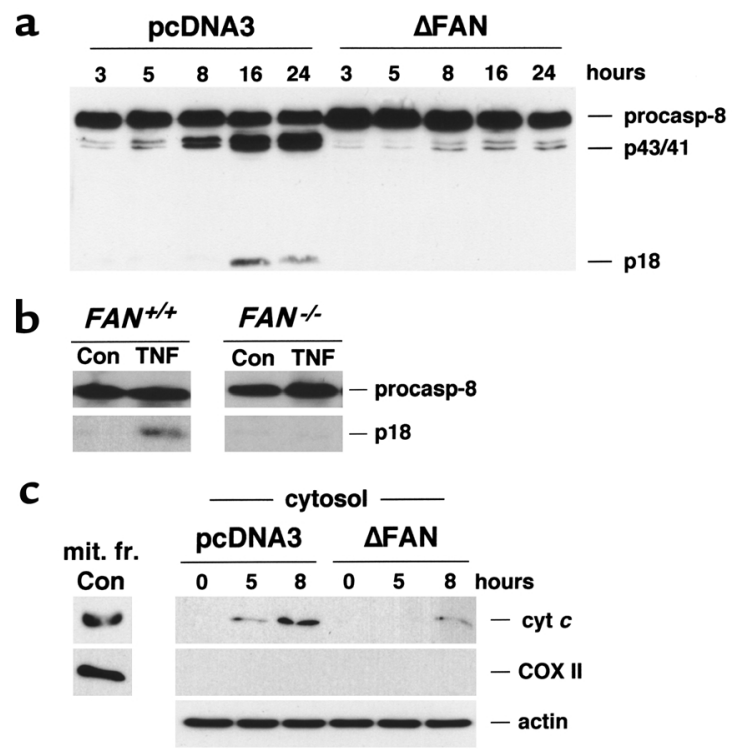

\section{Figure 7}

FAN is involved in TNF-induced processing of casp-8 and cyt $c$ release. Human fibroblasts transfected with empty vector (pcDNA3) or a vector encoding the mutated FAN ( $\triangle \mathrm{FAN}$ ) were incubated for the indicated times in the presence or absence of human TNF $(50 \mathrm{ng} / \mathrm{ml})$ and $50 \mu \mathrm{g} / \mathrm{ml}$ of cycloheximide. Murine cells were treated for 48 hours with TNF in serum-free medium. Casp-8 (a and b) processing (47) and cytosolic cyt $c$ accumulation (c) were examined by Western blot; actin and cytochrome oxidase (COXII) are shown as controls. A mitochondrial extract from untreated cells (mit. fr. Con) was used as a positive control for cyt $c$ and COX II. Migrations indicated are procasp- 8 , cleavage intermediate $p 43$ and $p 41$, active subunit $p 18$. The data are representative of three independent experiments. (which interacts only with CD120a) indicated that a FAN-null mutation impairs the CD120a-mediated response; (b) other TNF activities than cell death, such as $\mathrm{p} 42 / \mathrm{p} 44$ MAPK activation, NF- $\mathrm{KB}$ translocation, and CD5 4 expression, were unaffected by expression of the dominant negative form of FAN; and (c) the sensitivity to the anthracycline daunorubicin of human fibroblasts expressing the truncated FAN or of murine FAN-deficient cells was similar to that of their respective normal counterparts.

In human SV40-transformed fibroblasts, FAN is shown to inhibit TNF-induced cyt $c$ release into the cytosol, together with cleavage of effector casp-3 and 7. Surprisingly, FAN also impeded activation of initiator casp-8, and processing of its direct target, Bid. Casp8 is known to be potentially activated either at the level of the signaling complex generated by the recruitment to $\mathrm{CD} 120 \mathrm{a}$ of the $\mathrm{DD}$-containing proteins (e.g., TRADD and FADD) or downstream mitochondria (42). The comparable time course of activation observed for casp- 8 and casp- 3 suggests that, in transformed fibroblasts, casp- 8 is probably not at the apex of the protease cascade but could rather be processed indirectly. This is further substantiated by the fact that Bcl-2 overexpression, which blocks the death-related mitochondrial events, partially prevented TNF-induced apoptosis and processing of both casp-3 and casp-8 (B. Ségui et al., unpublished observations). These data might indicate that transformed fibroblasts behave as a type-II-like cells $(35,42)$.

Cer and TNF-induced apoptosis. Early studies identified the sphingolipid Cer as a potential second messenger in the signaling cascade initiated by TNF (reviewed in ref. 28). Cer generation was shown to result from the stimulation of an SMase activity and the subsequent hydrolysis of the membrane lipid SM. Through the activation of different downstream targets, Cer is believed to transduce various activities of TNF, including apoptosis $(27,29)$. Because TNF has been reported to activate two distinct SMases, i.e., the acid and neutral SMases (43), different biologic roles for Cer produced by these two enzymes have been suggested (13). With respect to the cytotoxic activity of TNF, two potential signaling mechanisms can now be proposed that link CD120a, Cer production, and induction of cell death.

On the one hand, the enhancement of an acid SMase activity, a pathway initiated at the DD and implicating the adapter proteins TRADD and FADD $(14,15)$, could result in the formation of Cer in acidic compartments (13). This Cer would activate the aspartyl-proteinase cathepsin D (44) that has been proposed to be involved in TNF-induced cell death (45). At least in human fibroblasts, this pathway does not seem to be critical for the death process, as cells lacking acid SMase (i.e., cells from patients with Niemann-Pick disease) are not protected from death induction by TNF (B. Ségui and T. Levade, unpublished observations). This pathway may, however, amplify the TNF response. On the other 
hand, Cer can be generated by a neutral, membranebound SMase through a pathway initiated at the NSD and involving FAN. This particular Cer might be an important contributor to the TNF-induced cell death process, given that we show here that obliteration of FAN function, which leads to impaired Cer generation, significantly inhibits apoptosis. Addition of exogenous Cer to FAN-deficient cells enables cell death induction, suggesting that Cer may mediate toxicity. Whether the production of Cer is the only mediator of FAN action (i.e., whether FAN can induce apoptosis independently of Cer), and what the molecular mechanisms linking Cer and cell death are, require further investigation.

Biologic functions of FAN. The original function of the adapter protein FAN is to serve TNF-induced stimulation of neutral SMase activity (19). Recent observations, as well as the present findings, have confirmed this role and enabled a better delineation of its activities. In contrast to initial speculation (19), FAN does not couple to $\mathrm{p} 42 / \mathrm{p} 44 \mathrm{MAPK}$ in TNF-stimulated cells (32) (see Figure 2). Moreover, activation of these MAPK, as well as the p38 kinase, is independent of the NSD but mediated by the DD of CD120a $(16,46)$. Activation of c-Raf-1 has also been proposed to be mediated by FAN (19), via a Cer-activated protein kinase, which then may link Cer to MAPK $(24,37)$. Two CD120a domains, including the FAN-interacting domain, may actually cooperate to activate c-Raf- 1 (38). Another example of interplay between different regions of CD120a has recently been published, indicating a role of the FAN-binding site in mediating actin reorganization in macrophages (41).

While the generation of FAN-deficient mice has revealed a function of FAN in TNF-mediated cutaneous barrier repair, it failed to demonstrate any crucial role in the regulation of immune and inflammatory responses (32). Previous studies (31) and the present studies indicate, however, that FAN helps controlling cytokine-induced (but not anthracycline-induced) apoptosis, as highlighted by the partial resistance of FAN-deficient fibroblasts to CD40- and CD120a-mediated cytotoxicity. The fact that obliteration of FAN functions in mouse has no major in vivo consequences (at least under the conditions tested) may indicate that some redundancy in the above-mentioned cytokine effects exists, or that FAN provides complementary signals for amplification of the response.

Altogether, the present data provide evidence for a novel, yet unrecognized role of the membrane-proximal region of CD120a and of the FAN adapter in TNF-mediated signaling of cell death. Our findings also emphasize the important notion of cooperative, rather than independent, effects of distinct regions of the TNF receptor in apoptosis signal transduction. With the exception of CD40 (31), it is still unknown whether FAN fulfills additional functions in the signaling of other members of the TNFR superfamily. In addition, further studies should clarify the molecular links between FAN, neutral SMase, and the cell death program.

\section{Acknowledgments}

We thank N. Andrieu-Abadie for discussion, and D. Nicholson, P. Krammer, G. Cohen, X. Wang, F. Tercé, and D. Perry for providing reagents. The technical assistance of S. Carpentier and M.A. Berges is gratefully acknowledged. This work was supported by grants from INSERM, Université P. Sabatier, VML and ARC. B. Ségui and O. Cuvillier are recipients of MESR and FRM, respectively.

1. Hengartner, M.O. 2000. The biochemistry of apoptosis. Nature.
407:770-776.

2. Ashkenazi, A., and Dixit, V.M. 1998. Death receptors: signaling and modulation. Science. 281:1305-1308.

3. Nagata, S. 1997. Apoptosis by death factor. Cell. 88:355-365.

4. Tracey, K.J., and Cerami, A. 1993. Tumor necrosis factor, other cytokines and disease. Annu. Rev. Cell Biol. 9:317-343.

5. Vandenabeele, P., Declercq, W., Beyaert, R., and Fiers, W. 1995. Two TNF receptors: structure and function. Trends Cell Biol. 5:392-399.

6. Wallach, D., et al. 1999. TNF receptor and Fas signaling mechanisms. Annu. Rev. Immunol. 17:331-367.

7. Chan, F.K., et al. 2000. A domain in TNF receptors that mediates ligand-independent receptor assembly and signaling. Science. 288:2351-2354.

8. Erickson, S.L., et al. 1994. Decreased sensitivity to TNF but normal Tcell development in TNF receptor-2-deficient mice. Nature. 372:560-563.

9. Pfeffer, K., et al. 1993. Mice deficient for the $55 \mathrm{kd} \mathrm{TNF}$ receptor are resistant to endotoxic shock, yet succumb to L. monocytogenes infection. Cell. 73:457-467.

10. Rothe, J., et al. 1993. Mice lacking the TNF receptor 1 are resistant to TNF-mediated toxicity but highly susceptible to infection by Listeria monocytogenes. Nature. 364:798-802.

11. Brakebusch, C., Nophar, Y., Kemper, O., Engelmann, H., and Wallach, D. 1992. Cytoplasmic truncation of the p55 TNF receptor abolishes signalling, but not induced shedding of the receptor. EMBO J. 11:943-950.

12. Tartaglia, L., Ayres, T., Wong, G., and Goeddel, D.V. 1993. A novel domain within the $55 \mathrm{kd}$ TNF receptor signals cell death. Cell. 74:845-853.

13. Wiegmann, K., Schütze, S., Machleidt, T., Witte, D., and Krönke, M. 1994. Functional dichotomy of neutral and acidic sphingomyelinases in TNF signaling. Cell. 78:1005-1015.

14. Wiegmann, K., et al. 1999. Requirement of FADD for TNF-induced activation of acid sphingomyelinase. J. Biol. Chem. 274:5267-5270.

15. Schwandner, R., Wiegmann, K., Bernardo, K., Kreder, D., and Krönke, M. 1998. TNF receptor death domain-associated proteins TRADD and FADD signal activation of acid sphingomyelinase. J. Biol. Chem. 273:5916-5922.

16. Boone, E., Vandevoorde, V., De Wilde, G., and Haegeman, G. 1998. Activation of $\mathrm{p} 42 / \mathrm{p} 44$ mitogen-activated protein kinases (MAPK) and p38 MAPK by TNF is mediated through the death domain of the $55-\mathrm{kDa}$ TNF receptor. FEBS Lett. 441:275-280.

17. Vandevoorde, V., Haegeman, G., and Fiers, W. 1997. Induced expression of trimerized intracellular domains of the human TNF p55 receptor elicits TNF effects. J. Cell Biol. 137:1627-1638.

18. Boldin, M.P., et al. 1995. Self-association of the "death domains" of the p55 TNF receptor and Fas/APO1 prompts signaling for TNF and Fas/APO1 effects. J. Biol. Chem. 270:387-391.

19. Adam-Klages, S., et al. 1996. FAN, a novel WD-repeat protein, couples the p55 TNF-receptor to neutral sphingomyelinase. Cell. 86:937-947.

20. Song, H.Y., Dunbar, J., Zhang, Y., Guo, D., and Donner, D.B. 1995. Identification of a protein with homology to hsp 90 that binds the type 1 TNF receptor. J. Biol. Chem. 270:3574-3581.

21. Boldin, M.P., Mett, I., and Wallach, D. 1995. A protein related to a proteasomal subunit binds to the intracellular domain of the p55 TNF receptor upstream to its 'death domain'. FEBS Lett. 367:39-44.

22. Adam, D., Wiegmann, K., Adam-Klages, S., Ruff, A., and Krönke, M. 1996. A novel cytoplasmic domain of the p55 TNF receptor initiates the neutral sphingomyelinase pathway. J. Biol. Chem. 1996:14617-14622.

23. Levade, T., Salvayre, R., and Douste-Blazy, L. 1986. Sphingomyelinases and Niemann-Pick disease. J. Clin. Chem. Clin. Biochem. 24:205-220.

24. Belka, C., et al. 1995. TNF- $\alpha$ activates $c$-raf- 1 kinase via the p55 TNF receptor engaging neutral sphingomyelinase. EMBOJ. 14:1156-1165.

25. Castellino, A.M., Parker, G., Boronenkov, I., Anderson, R., and Chao, M. 1997. A novel interaction between the juxtamembrane region of the p55 TNF receptor and phosphatidylinositol-4-phosphate 5-kinase. J. Biol. Chem. 272:5861-5870. 
26. De Vos, K., et al. 1998. The 55-kDa TNF receptor induces clustering of mitochondria through its membrane-proximal region. J. Biol. Chem. 273:9673-9680.

27. Obeid, L., Linardic, C., Karolak, L., and Hannun, Y. 1993. Programmed cell death induced by ceramide. Science. 259:1769-1771.

28. Hannun, Y., and Luberto, C. 2000. Ceramide in the eukaryotic stress response. Trends Cell Biol. 10:73-80.

29. Kolesnick, R., and Krönke, M. 1998. Regulation of ceramide production and apoptosis. Annu. Rev. Physiol. 60:643-665.

30. Andrieu, N., Salvayre, R., and Levade, T. 1994. Evidence against involvement of the acid lysosomal sphingomyelinase in the TNF- and IL-1 induced sphingomyelin cycle and cell proliferation in human fibroblasts. Biochem. J. 303:341-345.

31. Ségui, B., et al. 1999. CD40 signals apoptosis through FAN-regulated activation of the sphingomyelin-ceramide pathway. J. Biol. Chem. 274:37251-37258

32. Kreder, D., et al. 1999. Impaired neutral sphingomyelinase activation and cutaneous barrier repair in FAN-deficient mice. EMBO J. 18:2472-2479.

33. van Veldhoven, P., Matthews, T., Bolognesi, D., and Bell, R. 1992 Changes in bioactive lipids, alkylacylglycerol and ceramide, occur in HIV-infected cells. Biochem. Biophys. Res. Commun. 187:209-216.

34. Andrieu, N., Salvayre, R., Jaffrézou, J.P., and Levade, T. 1995. Low temperatures and hypertonicity do not block cytokine-induced stimulation

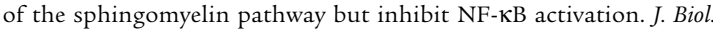
Chem. 270:24518-24524.

35. Cuvillier, O., Edsall, L., and Spiegel, S. 2000. Involvement of sphingosine in mitochondria-dependent Fas-induced apoptosis of type II Jurkat T cells. J. Biol. Chem. 275:15691-15700.

36. Jaffrézou, J.P., et al. 1996. Daunorubicin-induced apoptosis: triggering of ceramide generation through sphingomyelin hydrolysis. EMBO J 15:2417-2424

37. Yao, B., et al. 1995. Phosphorylation of Raf by ceramide-activated protein kinase. Nature. 378:307-310.

38. Hildt, E., and Oess, S. 1999. Identification of Grb2 as a novel binding partner of TNF receptor I. J. Exp. Med. 189:1707-1714.

39. Hess, S., and Engelmann, H. 1996. A novel function of CD40: induction of cell death in transformed cells. J. Exp. Med. 183:159-167.

40. Mackay, F., Rothe, J., Bluethmann, H., Loetscher, H., and Lesslauer, W. 1994. Differential responses of fibroblasts from wild-type and TNFR55-deficient mice to mouse and human TNF- $\alpha$ activation. J. Immunol. 153:5274-5284.

41. Peppelenbosch, M., et al. 1999. Multiple signal transduction pathways regulate TNF-induced actin reorganization in macrophages: inhibition of Cdc42-mediated filopodium formation by TNF. J. Immunol. 162:837-845.

42. Scaffidi, C., Kirchhoff, S., Krammer, P., and Peter, M. 1999. Apoptosis signaling in lymphocytes. Curr. Opin. Immunol. 11:277-285.

43. Levade, T., and Jaffrézou, J.P. 1999. Signalling sphingomyelinases: which, where, how and why? Biochim. Biophys. Acta. 1438:1-17.

44. Heinrich, M., et al. 1999. Cathepsin D targeted by acid sphingomyelinase-derived ceramide. EMBOJ. 18:5252-5263.

45. Deiss, L., Galinka, H., Berissi, H., Cohen, O., and Kimchi, A. 1996. Cathepsin $\mathrm{D}$ protease mediates programmed cell death induced by interferon-gamma, Fas/APO-1 and TNF- $\alpha$. EMBO J. 15:3861-3870.

46. Luschen, S., et al. 2000. Activation of ERK1/2 and cPLA(2) by the p55 TNF receptor occurs independently of FAN. Biochem. Biophys. Res. Commun. 274:506-512.

47. Sun, X.M., et al. 1999. Distinct caspase cascades are initiated in receptor-mediated and chemical-induced apoptosis. J. Biol. Chem. 274:5053-5060. 